\title{
Dietary and lifestyle patterns in UK postpartum women
}

\section{Abstract}

Introduction: Healthy dietary and physical activity behaviours during and after pregnancy are important for optimal maternal health. Unhealthy lifestyle habits promote excessive weight gain during pregnancy or weight retention in the postpartum period, which increase the risk of obesity. The aim of this study was to explore dietary habits and associated lifestyle patterns and barriers to healthy eating in UK postpartum women.

Methods: Cross-sectional study of 228 females (56.1\% were $25-34$ years old) with a mean postpartum period of $5.73 \pm 3.31$ months. Participants completed an online survey exploring eating habits, weight status, sleep duration, breastfeeding, nutrition knowledge, physical activity, provision of advice and barriers to healthy eating during the postpartum period. A sub-sample of 34 women (50\% of the sample were between 35-44 years old) completed an optional online dietary intake assessment using a multiple-pass 24-hour recall.

Results: In total, $73.7 \%$ were not meeting the five a day fruit and vegetable UK recommendations, $40.4 \%$ of women were skipping breakfast and $44.7 \%$ were skipping lunch every day. Average weight gain from pre-pregnancy to postpartum was $5.56 \pm 4.61 \mathrm{~kg}$ (range $=0.8$ to $25 \mathrm{~kg}$ ) with only $2.2 \%$ of women meeting current UK physical activity guidelines during the postpartum period. Consumption of high calorie snacks and meal skipping were significantly higher during the postpartum period compared to prepregnancy $(p<0.01)$. Women who were breastfeeding had significantly lower body mass index than those who were bottle feeding $(\mathrm{p}<0.05)$. Fatigue, lack of time and feeling stressed had the most impact on women's ability to eat healthily. Barriers to consume a balanced healthy diet were significantly greater for women with low combined household income and those having three or more children $(\mathrm{p}<0.05)$. Poor nutrition knowledge was significantly associated with increased meal skipping $(\mathrm{p}<0.05)$. The subsample dietary analysis $(\mathrm{N}=34)$ showed that women were not meeting the UK Dietary Reference Values for energy, fibre, iron, and vitamin D intakes. Those breastfeeding were not meeting requirements for calcium and zinc.

Discussion: To our knowledge, this is the first study to explore dietary habits and lifestyle patterns in UK postpartum women. Health care professionals should support women to adopt lifestyle behaviours following childbirth, with a greater focus on those likely to be experiencing more barriers. Advice given to women during the postpartum period needs to be tailored to potential differences in sociodemographic characteristics, pre-pregnancy health status and baseline nutrition knowledge and target multiple dietary and lifestyle behaviours.

\section{Conflict of Interest}

There is no conflict of interest 